\title{
La pasión de aprender: Un estudio correlacional entre los estilos de aprendizaje y el rendimiento académico
}

\section{The passion of learning: A correlational study between learning styles and academic performance}

\author{
A paixão pela aprendizagem: Um estudo de correlação entre estilos de \\ aprendizagem e desempenho académico
}

Jesús Yamid Redondo-Remolina*

\footnotetext{
a* Licenciado En Biología Y Química (UFPS), Especialista En Orientación Vocacional y Ocupacional (UFPS). Magister En Gerencia Educacional (UNY). Estudiante de doctorado en educación (UPEL-IPRGR), yamis.r@hotmail.com ORCID 0000-0002-9597-9033, Cúcuta, Colombia.
}

Forma de citar: Redondo, J. Y. (2018).La pasión de aprender: Un estudio correlacional entre los estilos de aprendizaje y el rendimiento académico. Perspectivas, 3(2), 34-45.

Recibido: Enero 15 de 2018

Aceptado: Junio 09 de 2018

\section{Palabras clave}

CHAEA

Estilos de aprendizaje

Pruebas saber 11

Rendimiento

Atcadémico
Resumen: El objetivo de este trabajo investigativo es analizar qué tipo de relación existe entre los estilos de aprendizaje y el rendimiento académico en las pruebas saber 11, en los estudiantes de educación media de la ciudad de Cúcuta. Esta investigación se ubica dentro del paradigma positivista con un enfoque cuantitativo y diseño descriptivo correlacional, se utilizó el instrumento Cuestionario Honey-Alonso De Estilos De Aprendizaje (CHAEA) y el rendimiento académico se midió con los resultados de las pruebas saber 11. La muestra fueron 133 estudiantes del grado 11, pertenecientes a las mejores instituciones oficiales de la ciudad, jornada de la mañana (categoría $\mathrm{A}+$ ). Como conclusión se determina que el estilo activo presenta una asociación significativa, de carácter negativo, con el rendimiento académico en las pruebas saber 11, de sociales y ciencias naturales a un nivel de significancia de 0,05 (bilateral). Por otra parte, el rendimiento académico en la prueba de ciencias naturales, presenta asociación significativa, con el estilo de aprendizaje reflexivo y teórico, con un nivel de significancia de 0,01 y 0,05 respectivamente. Se recomienda analizar otras variables posiblemente influyentes en el desempeño académico, como la motivación, prácticas pedagógicas entre otras.

\footnotetext{
*Autor para correspondencia Jesús Yamid Redondo Remolina yamis.r@hotmail.com
} 


\section{Keywords}

CHAEA

Learning styles

Tests know 11

Academic performance

\section{Palavras chave}

CHAEA

Estilos de aprendizagem

Testes sabem 11

Desempenho acadêmico
Abstract: The objective of this research work is to analyze what type of relationship exists between learning styles and academic performance in the tests known as 11 , in middle school students in the city of Cúcuta. This research is located within the positivist paradigm with a quantitative approach and descriptive correlational design, the instrument HoneyAlonso Questionnaire of Learning Styles (CHAEA) was used (and the academic performance was measured with the results of the tests know 11. The sample There were 133 students of grade 11, belonging to the best official institutions of the city, morning shift (category A +) .In conclusion, it was determined that the active style presents a significant association, of a negative nature, with the academic performance in the tests know 11, of social and natural sciences at a level of significance of 0.05 (bilateral) On the other hand, the academic performance in the natural science test, presents significant association, with the reflective and theoretical learning style, with a level of significance of 0.01 and 0.05 respectively, it is recommended to analyze other potentially influential variables in the Academic performance, such as motivation, pedagogical practices among others.

Resumo: O objetivo desta pesquisa é analisar que tipo de relação entre estilos de aprendizagem e desempenho acadêmico em testes ou seja, 11, estudantes do ensino médio na cidade de Cucuta. Esta pesquisa está dentro do paradigma positivista, com abordagem quantitativa e design correlacional descritiva Questionário Honey-Alonso estilos de aprendizagem (CHAEA) instrumento (e desempenho acadêmico foi usada foi medida com os resultados do teste para saber 11. Amostra foram 133 alunos em grau 11, pertencentes às melhores instituições oficiais da cidade, dia de manhã (categoria a + ). em conclusão, é determinado que o estilo ativo apresenta uma associação significativa, caráter negativo, o desempenho acadêmico em testes ou seja, 11 de ciências sociais e naturais a um nível de significância de 0,05 (bilateral). por outro lado, teste de desempenho acadêmico em ciências naturais, apresenta associação significativa com o estilo de aprendizagem teórica e reflexiva, com nível de significância de 0,01 e 0,05 , respectivamente, recomenda-se analisar outras variáveis potencialmente influentes no Desempenho acadêmico, como motivação, práticas pedagógicas entre outros. 


\section{Introducción}

El aprendizaje es un elemento esencial para la vida humana, es un fenómeno que se produce desde el comienzo de la vida. Cuando se piensa en el entorno escolar, la comprensión de cómo el aprendizaje se lleva a cabo y cuáles son los fenómenos que intervienen en este proceso de convertirse en aspectos cruciales de la escuela hacia el cumplimiento de su función, que es proporcionar el máximo desarrollo de los estudiantes. Como ser único, cada estudiante asimila el aprendizaje a su manera y aprende de modo exitoso cuando la información adquirida se almacena y se procesa, para luego producir con eficiencia. Esto hace que los aprendices adopten sus propias estrategias, porque son las que les permiten dar resultados satisfactorios de aquello que han adoptado y personalizado, siendo estos los aspectos guiadores en la producción del conocimiento.

El concepto de estilos de aprendizaje, es establecido por diferentes definiciones, en la actualidad hay un gran número de autores que han aportado su definición. En general se plantea como las distintas formas de que un sujeto tiene acceso al aprendizaje. Sin embargo, también hay diferencias fundamentales que tienen relación con el modelo de aprendizaje que plantee el autor, que va desde cómo es captada la información por los sentidos, hasta como es intervenida y procesada por los individuos.

De acuerdo a estas consideraciones, Alonso y otros investigadores en 1994 integraron diferentes conceptos, en especial los de Keefe J. (1988), y definen los estilos de aprendizaje "como los rasgos cognitivos, afectivos y fisiológicos, que sirven como indicadores relativamente estables, de cómo los discentes perciben, interrelacionan y responden a sus ambientes de aprendizaje" (p. 14). Por otra parte, Guild y Garger (1998), manifiestan que "otros autores consideran que el término estilo se comenzó a utilizar por los investigadores a partir del siglo XX en concreto por aquéllos que trabajaron en distinguir las diferencias entre las personas en áreas de la psicología y de la educación" (p. 61). Ahora bien, Lozano (2000) después de analizar diversas teorías y de integrar múltiples conceptos anteriores definió estilo como "un conjunto de preferencias, tendencias y disposiciones que tiene una persona para hacer algo y que se manifiesta a través de un patrón conductual y de distintas fortalezas que 10 hacen distinguirse de los demás". (p. 17). En este sentido, Castaño y Calles (2006) definen "los estilos de aprendizaje como las preferencias personales a la hora de procesar información y enfrentarse a una tarea de aprendizaje en distintos contextos". (p. 26).

Dadas las condiciones que anteceden, Alonso C. (1992) manifiesta que a causa del crecimiento del número de "teorías de y definiciones de aprendizaje de manera proporcional ha aumentado los modelos de estilos de aprendizaje. Un ejemplo es como Honey y Mumford (1986), describió los estilos de aprendizaje Activos, Reflexivos, Teóricos y Pragmáticos en base a la teoría de Kolb” (p. 12)

Dadas las anteriores definiciones se puede ratificar la apreciación hecha por Varela H, (2014) en la que expresa que "es necesario, identificar las necesidades del estudiante, escuchar sus ideas, estimular sus experiencias con la intención de que éste se apropie de sus problemáticas, y así lograr que su aprendizaje sea significativo. (p. 51). En este sentido, los aportes hechos por los autores están centrados en tomar las experiencias o aprendizajes propios como mecanismo para resolver las exigencias del medio, de igual forma se orientan hacia la caracterización del individuo en sus aspectos emocionales, sociales y físicos. Buscando enfatizar en el papel que deben tener los mediadores docentes, los compañeros de estudio y la familia en el proceso de interrelación del estudiante con su ambiente, para que pueda desarrollar aspectos referentes a su personalidad, satisfaciendo sus necesidades intelectuales, afectivas y sociales, enfrentándolo a nuevos estímulos y diferentes retos que le permitan hallar soluciones viables a las demandas del entorno, como se mencionó anteriormente.

Ahora bien, la preocupación con la identificación de los factores que afectan el rendimiento del estudiante ha sido desde hace mucho tiempo el 
tema de la investigación científica en diversas áreas. Es por ello, que al conocer los estilos de aprendizaje se puede contribuir a la comprensión de los procesos de aprendizaje, incluso en los entornos virtuales de enseñanza. Por tal motivo, este proyecto surge como marco iniciador de un proceso de investigación acerca de las variables que pueden influenciar el rendimiento académico en educación media siendo una de las variables influyentes en la deserción escolar HernándezDávila \& Díaz-Abdala (2017), teniendo como variables a relacionar los estilos de aprendizaje y el rendimiento, en donde se pretende desarrollar una caracterización educativa (estudiantes), teniendo en cuenta los mejores resultados de las pruebas saber 11 y a la luz del momento histórico que vive la educación media cucuteña (con el posicionamiento de más 11 colegios con nivel $\mathrm{A}+$ de los cuales seis son públicos) de esta manera se podrá configurar el clima y cultura organizacional de calidad de las mejores instituciones oficiales de la ciudad de Cúcuta. Esta investigación sigue la línea del trabajo realizado por Hernández, Prada \& Rincón (2018) dónde se consideró la relación entre las inteligencias múltiples y el rendimiento académico. Con ello se podrá promover, adoptar o diseñar las posibles vías de explicación que respondan al fenómeno de los factores educacionales incidentes en el mejoramiento del desempeño académico y conducente al mejoramiento de la calidad educativa.

\section{Materiales y métodos}

\section{Diseño}

Estudio de investigación de tipo cuantitativo con un diseño descriptivo y correlacional, no experimental transeccional. Es un estudio correlacional pues pretende medir el grado de relación que existe entre las dos variables: Estilos de aprendizaje y el rendimiento académico (en un contexto en particular). En este caso el diseño (bajo un enfoque no experimental) es el transeccional ya que se recolectan datos en un solo momento, en un tiempo único. Su propósito es describir variables y analizar su interrelación en un momento dado.

\section{Participantes}

Se tomó una muestra no probabilística por conveniencia, teniendo en cuenta los datos suministrados por la Revista Dinero (2016) y el Grupo Educativo Milton Ochoa (2016). En este sentido, se seleccionaron los estudiantes del grado once de las Instituciones Educativas Oficiales con los mejores promedios de las pruebas saber 11 año 2016, jornada de la mañana, categoría $\mathrm{A}+$, con una totalidad de 133 estudiantes, pertenecientes al Instituto Técnico Nacional De Comercio (ITNC) con 60 estudiantes, el Colegio Sagrado Corazón de Jesús (CORSAJE) con 33 estudiantes y la Institución Educativa Colegio Santos Apóstoles (COLSANAP) con 40 estudiantes. El 47\% de la muestra está compuesta por mujeres, mientras el $53 \%$ son hombres. Las edades de la muestra están en un rango de 15 a 20 años de edad, con una media (M) 16,68 años y una desviación estándar (DE) de ,847 y varianza (v) de ,718. E1 44\% de la muestra tiene una edad de 16 años (59 estudiantes) y $41 \%$ tiene 17 años (54 educandos), tres estudiantes tienen 15 años (2\% de la muestra) y 12 jóvenes 18 años ( $9 \%$ de la muestra); 4 estudiantes tienen 19 años y un solo joven con 20 años.

\section{Instrumento}

La recolección de los datos de la variable de Estilos de Aprendizaje, fue analizada a través de la aplicación del Cuestionario Honey Alonso sobre Estilos de Aprendizaje (CHAEA). El índice de confiabilidad se calculó por medio de la fórmula, propuesta en 1937 por Kuder- Richardson (KR20), que es recomendada para medir la consistencia interna de una escala cuando los ítems tienen respuestas dicotómicas. Respecto a la confiabilidad instrumental, se encontró que su índice de consistencia interna KR-20 es aceptable, (activo $\mathrm{KR}-20=.68$, reflexivo KR-20 $=.74$, teórico KR- 
$20=.64$ y pragmático KR-20 $=.62$ ). La magnitud de esta consistencia es similar a la reportada por Alonso et al. (1997) "(activo $\alpha=.62$, reflexivo $\alpha=$ .72 , teórico $\alpha=.65$ y pragmático $\alpha=.58$ )" (p. 80), por Camarero et al. (2000) "(activo $\alpha=.68$, reflexivo $\alpha=.64$, teórico $\alpha=.63$ y pragmático $\alpha=.59$ )" (p. 622).

La validación de contenido, fue realizada a juicio de tres expertos, denotando la necesidad de realizar adaptaciones a los reactivos del instrumento. (Items 4, 5, 7, 17, 23, 31, 59, 63, 66, 75)

\section{Resultados y discusiones}

\section{Descripción de la variable de estudio: Estilos de aprendizaje}

En la tabla 1, se puede apreciar que los estilos de aprendizaje activo y reflexivo, tienen en algunos casos la puntuación máxima, a diferencia del estilo teórico y pragmático que no lo logran. Pero estos últimos presentan mejores puntuaciones mínimas respecto a los otros dos estilos de aprendizaje.

Tabla 1. Estadística descriptiva de los estilos de aprendizaje

\begin{tabular}{ccccccc}
\hline \multicolumn{7}{c}{ Estadísticos descriptivos } \\
\hline & $\mathbf{N}$ & Mínimo & Máximo & Media & $\begin{array}{c}\text { Desviación } \\
\text { estándar }\end{array}$ & Varianza \\
Activo & 133 & 4 & 20 & 11,75 & 3,456 & 11,946 \\
Reflexivo & 133 & 4 & 20 & 14,80 & 3,118 & 9,719 \\
Teórico & 133 & 6 & 19 & 12,87 & 2,737 & 7,491 \\
$\begin{array}{c}\text { Pragmático } \\
\text { N válido (por }\end{array}$ & 133 & 5 & 19 & 13,17 & 2,580 & 6,654 \\
lista) & 133 & & & & & \\
\hline
\end{tabular}

De acuerdo con los resultados, los estudiantes tienen mayores puntuaciones hacia el estilo de aprendizaje reflexivo con una media de 14,80, seguido del pragmático (media de 13,17), teórico (media de 12,87) y activo (media de 11,75), todos con una preferencia moderada, aplicando el baremo general. En la figura I, se muestra la tendencia promedial de la muestra hacia el estilo de aprendizaje reflexivo.

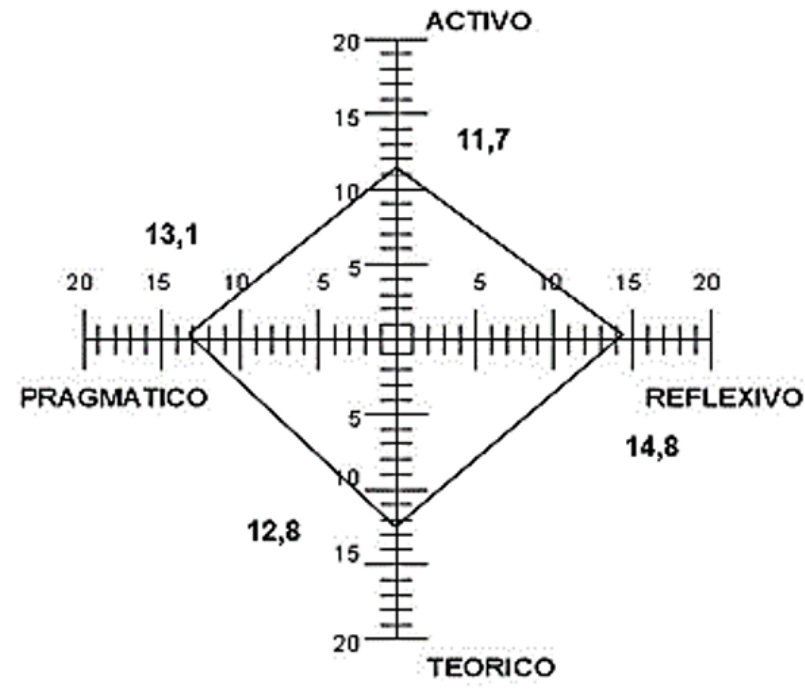

Figura 1. Estilos de aprendizaje de toda muestra Fuente: Autor

Los resultados del Tabla 2, manifiestan que el estilo reflexivo obtuvo la media más alta con relación a los estilos, pragmático, teórico y activo siendo este último el de menor promedio con relación a la media. En el ITNC, al igual que el CORSAJE, el estilo reflexivo tuvo la mejor media, a diferencia del COLSANAP en donde lo educandos puntuaron mejor en el estilo de aprendizaje pragmático.

Tabla 2. Estilos de aprendizaje por instituciones educativas

\begin{tabular}{ccccc}
\hline $\begin{array}{c}\text { Estilos de } \\
\text { aprendizaje / } \\
\text { Instituciones } \\
\text { educativas }\end{array}$ & $\begin{array}{c}\text { Instituto } \\
\text { Técnico } \\
\text { Nacional De } \\
\text { Comercio }\end{array}$ & $\begin{array}{c}\text { Institución } \\
\text { Educativa } \\
\text { Colegios } \\
\text { Santos } \\
\text { Apóstoles }\end{array}$ & $\begin{array}{c}\text { Colegio } \\
\text { Sagrado } \\
\text { Corazón } \\
\text { Jesús }\end{array}$ & $\begin{array}{c}\text { Promedio } \\
\text { general }\end{array}$ \\
\hline Activo & 11,06 & 12,72 & 11,81 & 11,75 \\
Reflexivo & 15,68 & 12,97 & 15,42 & 14,80 \\
Teórico & 13,13 & 12,20 & 13,21 & 12,87 \\
Pragmático & 13,43 & 13,15 & 12,69 & 13,17 \\
\hline
\end{tabular}

El gráfico 1, cada una de las instituciones educativas tiene una preferencia moderada, por cada uno de los estilos de aprendizaje, exceptuando la Institución Educativa Colegios Santos Apóstoles, que tiene una preferencia baja en el estilo de aprendizaje reflexivo. Por tanto, las instituciones son homogéneas respecto a esta variable. 


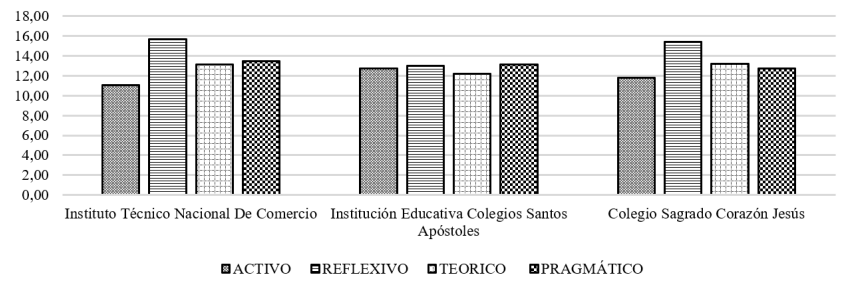

Gráfica 1. Promedios de estilos de aprendizaje por instituciones educativas

De acuerdo a la tabla 3, el 54\% de la muestra tiene una preferencia moderada por estilo de aprendizaje reflexivo (es el porcentaje más alto respecto en esa preferencia). Por otra parte, solo un $1 \%$ tiene preferencia muy alta en ese estilo, en contraste con el $23 \%$ el $18,8 \%$ y el $17,3 \%$, del estilo activo, teórico, y pragmático, respectivamente. Lo que indica que existe mayor población con preferencia alta y muy alta por los demás estilos. Ya que los estudiantes pueden puntuar de forma diferente para cada una de los estilos, lo idóneo sería que tuvieran una preferencia alta o muy alta por cada uno de los estilos, indicando que no tendría una tendencia de aprendizaje marcada y su aprendizaje sería mejor, sin tener en cuenta el estilo de enseñanza.

Tabla 3. Frecuencias de los estilos de aprendizaje

\begin{tabular}{|c|c|c|c|c|c|c|c|c|c|c|}
\hline \multirow[t]{2}{*}{$\begin{array}{c}\text { Estilos de } \\
\text { aprendizaje }\end{array}$} & \multicolumn{2}{|c|}{$\begin{array}{c}\text { Preferencia } \\
\text { Muy Baja }\end{array}$} & \multicolumn{2}{|c|}{$\begin{array}{c}\text { Preferencia } \\
\text { Baja }\end{array}$} & \multicolumn{2}{|c|}{$\begin{array}{c}\text { Preferencia } \\
\text { Moderada }\end{array}$} & \multicolumn{2}{|c|}{$\begin{array}{c}\text { Preferencia } \\
\text { Alta }\end{array}$} & \multicolumn{2}{|c|}{$\begin{array}{c}\text { Preferencia } \\
\text { Muy Alta }\end{array}$} \\
\hline & $\mathbf{F}$ & $\%$ & $\mathbf{F}$ & $\%$ & $\mathbf{F}$ & $\%$ & $\mathbf{F}$ & $\%$ & $\mathbf{F}$ & $\%$ \\
\hline Activo & 9 & $6,8 \%$ & 15 & $11,3 \%$ & 55 & $41,4 \%$ & 23 & $17,3 \%$ & 31 & $23,3 \%$ \\
\hline Reflexivo & 12 & $9 \%$ & 24 & $18 \%$ & 72 & $54,1 \%$ & 24 & $18 \%$ & 1 & $8 \%$ \\
\hline Teórico & 2 & $1,5 \%$ & 13 & $9,8 \%$ & 57 & $42,9 \%$ & 36 & $27,1 \%$ & 25 & $18,8 \%$ \\
\hline Pragmático & 6 & $4,5 \%$ & 15 & $11,3 \%$ & 49 & $36,8 \%$ & 40 & $30,1 \%$ & 23 & $17,3 \%$ \\
\hline F: Frecuencia & & Porcen & & & & & & & & \\
\hline
\end{tabular}

Análisis y Pruebas Estadísticas para Diferencias por Variables Demográficas: Comparaciones por Sexo

Los puntajes promedios, del gráfico 2, indican que los hombres tuvieron mayores puntuaciones en los estilos de aprendizaje, activo, teórico y pragmático. Las mujeres obtuvieron una media más alta en el estilo de aprendizaje reflexivo.

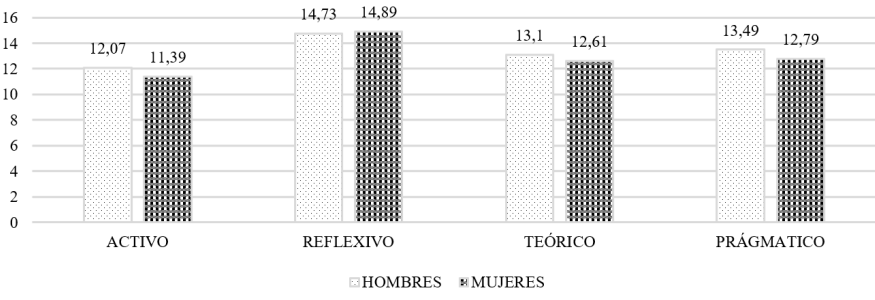

Gráfica 2. Puntajes de estilos de aprendizaje por sexo

\section{Prueba De Muestras Independientes Por Género: Estilos de aprendizaje}

Mediante Prueba de Levene de igualdad de varianzas, se afirma que el P-Valor $>\alpha(0,05)$, por tanto, se acepta que las varianzas son iguales. En este sentido, la prueba T De Student Para Muestras Independientes, expresa que no se obtienen diferencias considerables en relación con el género. De acuerdo con los resultados derivados del SPSS versión 24, no se observa ningún resultado inferior a $\alpha$ (0,05), (Activo: F: 1,170 Sig: ,281, Reflexivo: F: ,093 Sig: ,761, Teórico: F ,861 Sig: ,355, Prágmatico: F: ,036 Sig: ,849). De acuerdo con los resultados, no se observa ningún valor inferior a $(0,05)$.

Se aplicó la prueba Anova de un factor para hallar posibles diferencias por edades. (Activo: F: ,237 Sig: ,945, Reflexivo: F: ,774 Sig: ,570, Teórico: F: ,604 Sig: ,697, Prágmatico: F: ,244 Sig: ,942) Acorde a los resultados de la prueba, con un $95 \%$ de confianza, se puede afirmar que no existen diferencias significativas por edades en los constructos principales que conforman la variable de estilos de aprendizaje (Activo, reflexivo, teórico, pragmático) ya que $\mathrm{p} \geq 0,05$.

\section{Descripción de la variable de estudio: Rendimiento académico}

La variable rendimiento académico fue medida con las pruebas estandarizadas Saber 11, diseñadas por el Instituto Colombiano para la Evaluación de la Educación (ICFES) para medir la calidad de la educación.

De acuerdo a la tabla 4, la mejor institución educativa oficial según los resultados de las 
pruebas saber 11, es el Instituto Técnico Nacional De Comercio (ITNC), con un promedio general de 67,06. En este sentido la institución obtuvo el mejor promedio en las competencias de lectura crítica, matemáticas, ciencias naturales e inglés. El segundo mejor promedio lo obtuvo el Colegio Sagrado Corazón De Jesús (CORSAJE) quien solo logró superar promedialmente en la prueba de sociales, al ITNC. La Institución Educativa Colegio Santos Apóstoles (COLSANAP), no obtuvo promedios superiores en ninguna de las competencias respecto a las otras dos instituciones educativas.

Tabla 4. Promedio General Pruebas Saber 11 Instituciones Educativas

\begin{tabular}{cccc}
\hline & $\begin{array}{c}\text { Instituto Técnico Nacional } \\
\text { De Comercio }\end{array}$ & $\begin{array}{c}\text { Institución Educativa Colegios } \\
\text { Santos Apóstoles }\end{array}$ & $\begin{array}{c}\text { Colegio Sagrado Corazón } \\
\text { Jesús }\end{array}$ \\
\hline Lectura Critica & 65,5 & 62,7 & 63,4 \\
Matemáticas & 70,4 & 65,9 & 69,6 \\
Sociales & 63,9 & 61,5 & 64,7 \\
Ciencias & 68,8 & 61,9 & 64,6 \\
Naturales & 66,7 & 62,2 & 66,4 \\
Ingles & 67,06 & 62,84 & 65,74 \\
Promedio & General & &
\end{tabular}

De acuerdo al grafico 3, la competencia de matemáticas tuvo el mejor desempeño con un puntaje promedio de 68,9 , seguido de Ciencias Naturales con 65,6, Ingles con 65,3, lectura crítica 64,1 y por último Ciencias Sociales con un puntaje $63,4$.

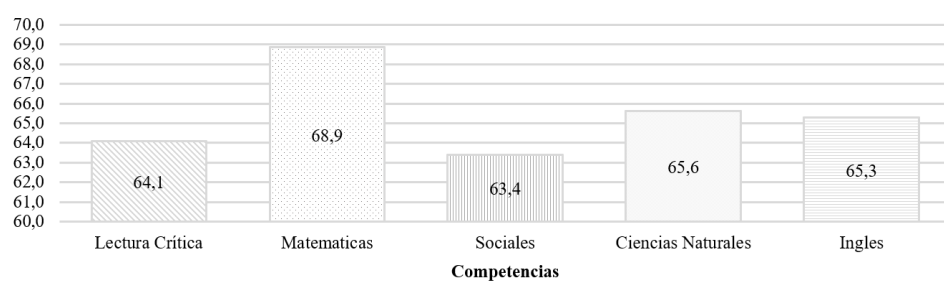

Gráfica 3. Promedios por áreas de competencia

El grafico 4, indica que el nivel de desempeño 1 , se encuentra ausente en las cuatro áreas de competencia. Este hace referencia a que el estudiante, no supera las preguntas de menor complejidad de las pruebas del examen. El nivel de desempeño 2, se encuentra ausente en la competencia de lectura crítica y matemáticas, pero con presencia alta del $74,4 \%$ en la competencia de sociales y mínima del
$6 \%$ en ciencias Naturales. Este indica que supera las preguntas de menor complejidad de cada prueba del examen.

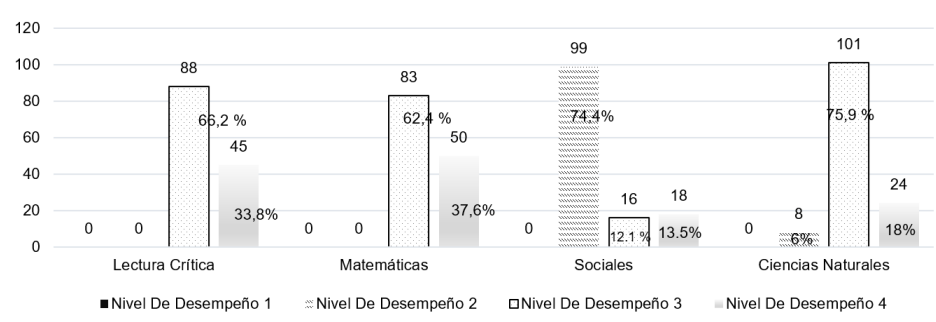

Gráfica 4. Niveles de desempeño: Competencia de lectura crítica, matemáticas, sociales y ciencias naturales

El nivel de desempeño 3, tiene frecuencias y porcentajes similares en las competencias de lectura crítica, matemáticas y una diferencia más notable en ciencias naturales, con valores porcentuales de $66,2 \%, 62,4 \%$ y $75,9 \%$ respectivamente. Este hace referencia a que el estudiante muestra un desempeño adecuado en las competencias exigibles para las pruebas del examen, este es el nivel esperado que todos o la gran mayoría de los estudiantes deberían alcanzar. El nivel de desempeño 4, manifiesta que el educando muestra un desempeño sobresaliente en las competencias esperadas en cada prueba del examen. El valor porcentual más alto en este nivel los obtuvo la prueba de matemáticas con $37,6 \%$, seguido de lectura crítica con $33,8 \%$, ciencias naturales con $18 \%$ y sociales con $13,5 \%$. Es preciso mencionar que estos cuatro niveles de desempeño, solo aplican para las pruebas de lectura crítica, matemáticas, ciencias naturales y sociales, ya que para la prueba de inglés hay otros niveles de desempeño estipulados y categorizados.

El grafico 5, manifiesta que el nivel de desempeño A2, es el más representativo de la muestra con una frecuencia de 93 estudiantes abarcando el $70 \%$ muestral, este nivel indica que los estudiantes son capaces de comprender frases y expresiones de uso frecuente relacionadas con áreas de experiencia especialmente relevantes (información básica sobre él mismo y su familia, compras, lugares de interés, ocupaciones, etcétera). 


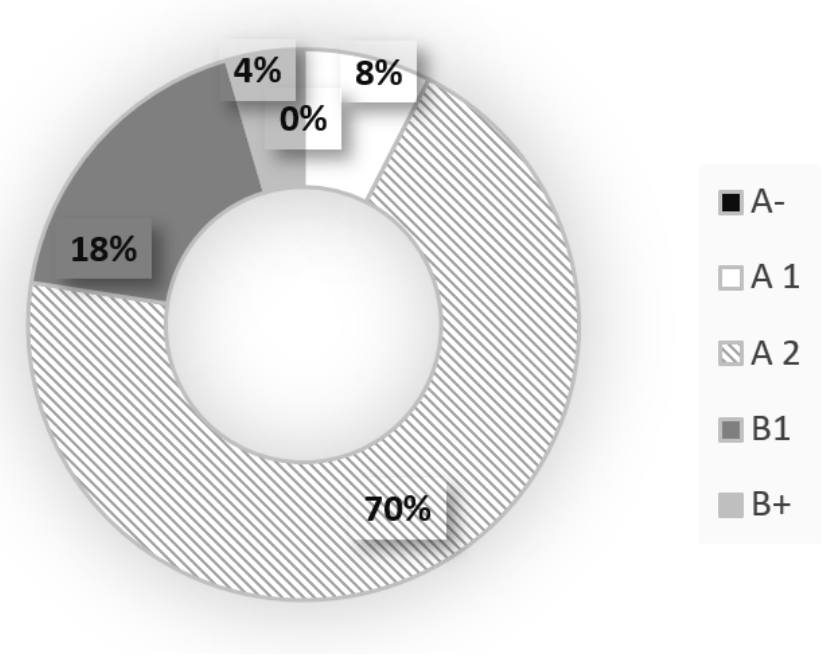

Gráfica 5. Niveles de desempeño: Competencia de inglés

El nivel B1, nos hace referencia a que el estudiante es capaz de comprender y utilizar expresiones cotidianas de uso muy frecuente, así como frases sencillas destinadas a satisfacer necesidades inmediatas. Puede reconocer el lenguaje adecuado para pedir y dar información personal básica sobre su domicilio, sus pertenencias y las personas que conoce, en este nivel solo están 24 estudiantes (18\%).

En el nivel A1, solo están 10 jóvenes (8\%), en este nivel el estudiante es capaz de comprender los puntos principales de textos claros y en lengua estándar, si estos tratan cuestiones conocidas, ya sea en situaciones de trabajo, de estudio o de ocio. El educando puede identificar la descripción de experiencias y acontecimientos. En el nivel B+, solo 6 educandos (4\%), superan las preguntas de mayor complejidad de la prueba. Es perentorio mencionar que el nivel A- se encuentra ausente. De acuerdo a la Tabla 5, y siguiendo la línea de los mejores promedios de las pruebas saber 11, el Instituto Técnico Nacional De Comercio, aporta 20 estudiantes a los posibles beneficiarios de las becas ser pilo paga, con un 15\% de la muestra total. Por otra parte, los Colegios Santos Apóstoles y Sagrado Corazón De Jesús, tienen un aporte igual con 7 educandos, respectivamente.

Tabla 5. Frecuencias de las becas por Institución Educativa

\begin{tabular}{cccccccccc}
\hline & \multicolumn{3}{c}{$\begin{array}{c}\text { Instituto Técnico Nacional De } \\
\text { Comercio }\end{array}$} & \multicolumn{2}{c}{$\begin{array}{c}\text { Institución Educativa Colegios } \\
\text { Santos Apóstoles }\end{array}$} & \multicolumn{3}{c}{$\begin{array}{c}\text { Colegio Sagrado Corazón } \\
\text { Jesús }\end{array}$} \\
\hline & $\mathrm{F}$ & $\% \mathrm{I}$ & $\% \mathrm{~T}$ & $\mathrm{~F}$ & $\% \mathrm{I}$ & $\% \mathrm{~T}$ & $\mathrm{~F}$ & $\% \mathrm{I}$ & $\% \mathrm{~T}$ \\
Posibles becados & 20 & 33,3 & 15.0 & 7 & 17,5 & 5,2 & 7 & 20,6 & 5,2 \\
Sin becas & 40 & 66.6 & 30,1 & 33 & 82,5 & 25,0 & 26 & 79,4 & 19,5 \\
Total & 60 & 100 & 45,1 & 40 & 100 & 30,2 & 33 & 100 & 24,7 \\
\hline
\end{tabular}

F: Frecuencia \%I: Porcentaje Institucional \%T: Porcentaje Total de la muestra

\section{Prueba De Muestras Independientes Por Género: Rendimiento Académico}

Mediante Prueba de Levene de igualdad de varianzas, se afirma que el $p>\alpha$, por tanto, se acepta que las varianzas son iguales. En este sentido, la prueba T De Student Para Muestras Independientes, manifiesta 
que no se obtienen diferencias considerables en relación con el género. (Lectura Crítica: F: ,362; Sig: ,546; Matemáticas: F: ,112 Sig: ,740; Sociales: F: 1,91 Sig: ,246; Ciencias Naturales: F: ,132 Sig: ,670; Inglés: F: 2,03 Sig: ,122). De acuerdo con los resultados derivados del SPSS versión 24 , no se observa ningún resultado inferior a $\alpha(0,05)$.

Al aplicar la prueba ANOVA, con un 95\% de confianza, se puede afirmar que no existen diferencias significativas por edades en las competencias saber de inglés, matemáticas y ciencias naturales, ya que $\mathrm{p}>0,05$. (Lectura Crítica: $F: 2,7$ Sig: ,022\%; Matemáticas: F: 2,0 Sig: ,073; Sociales: F: 3,1 Sig: ,010*; Ciencias Naturales: F: 1,1 Sig: ,358; Inglés: F: ,6 Sig: ,669). Por otra parte, sólo se deben considerar significativas las diferencias por edades para las competencias de lectura crítica y ciencias sociales.

\section{Análisis de correlación entre los estilos de aprendizaje y el rendimiento académico}

\section{Preliminares}

El objetivo de esta fase de la investigación es el de estudiar la relación la motivación académica total y el rendimiento académico basado en los resultados de las pruebas saber 11. Tal y como ya se ha puntualizado, el uso del coeficiente de correlación de Pearson exige la satisfacción de cinco premisas: (1) nivel de medición de intervalo o razón (2) observaciones en pares, (3) ausencia de datos atípicos, (4) linealidad y (5) normalidad bivariada. El cumplimiento de los dos primeros postulados se establece a partir de consideraciones teóricas: las variables de ambas características han sido definidas como puntajes, de modo que pueden tratarse como variables medidas en escala de intervalo, incluso de razón si el sentido de esta apreciación es todavía más amplio. A su vez, los datos han sido obtenidos en forma de pares ordenados (xi, yi), donde xi y yi representan el resultado de encuestar al i-ésimo sujeto con respecto a las dimensiones X y Y. Ahora bien, la satisfacción de los supuestos de normalidad y linealidad, así como la identificación de valores atípicos sí requiere de procedimientos numéricos o gráficos para su comprobación. En tal sentido, se ha ejecutado la siguiente rutina:

Paso 1: Se ejecutó un análisis exploratorio bivariado para establecer la presencia de datos atípicos. El criterio empleado ha sido el del valor-P bajo la distribución Ji-Cuadrado para las distancias de Mahalanobis de cada observación. La identificación de estos se dio cuando el nivel de significación observado fue menor que .001. Los registros que resultaron aberrantes fueron descartados del conjunto luego de comparar los coeficientes de correlación, antes y después de la extracción. Cambios significativos en los valores de tales índices sustentaron la eliminación del caso.

Paso 2: Se llevó a cabo un análisis de normalidad bivariado y multivariado, mediante las pruebas de Mardia, Henze-Zirkler y Royston, utilizando el software $r$ studio. La normalidad se asumió cuando por lo menos dos de los tres contrastes resultaron no significativos.

Paso 3: Se constató la linealidad mediante gráficos de dispersión bivariados y mediante la significación del coeficiente de correlación de Pearson. No se observó linealidad en ninguno de los casos. Los resultados de esta parte del trabajo pueden apreciarse en la Tabla 6. 
Tabla 6. Evaluación de los supuestos necesarios para usar el coeficiente de correlación de Pearson entre los estilos de aprendizaje y el rendimiento académico

\begin{tabular}{|c|c|c|c|c|c|c|c|c|c|c|}
\hline Comb. & Medición & Elim. & $R$ antes & $R$ desp. & Lin. & Mardia & Royston & H-Z & Norm. & Conclusión \\
\hline $\mathrm{C} 01$ & Intervalo & 1 & $-0,144$ & $-0,085$ & No & .52909 & .00001 & .08930 & $\mathrm{Si}$ & Spearman \\
\hline $\mathrm{C} 02$ & Intervalo & 0 & 0,282 & 0,023 & No & .52088 & .00040 & .01235 & No & Spearman \\
\hline $\mathrm{C} 03$ & Intervalo & 1 & 0,194 & 0,272 & No & .00640 & .00001 & .00002 & No & Spearman \\
\hline C04 & Intervalo & 1 & 0,015 & 0,109 & No & .00005 & .00030 & .00003 & No & Spearman \\
\hline $\mathrm{C} 05$ & Intervalo & 0 & 0,352 & 0,342 & No & .00013 & .00191 & .00008 & No & Spearman \\
\hline $\mathrm{C} 06$ & Intervalo & 0 & 0,158 & 0,149 & No & .00004 & .00007 & .00003 & No & Spearman \\
\hline $\mathrm{C} 07$ & Intervalo & 1 & $-0,123$ & $-0,043$ & No & .00069 & .00008 & .00040 & No & Spearman \\
\hline $\mathrm{C} 08$ & Intervalo & 0 & 0,086 & 0,058 & No & .00070 & .00020 & .00005 & No & Spearman \\
\hline C09 & Intervalo & 0 & 0,140 & 0,106 & No & .00060 & .00005 & .00046 & No & Spearman \\
\hline $\mathrm{C} 10$ & Intervalo & 0 & $-0,351$ & $-0,340$ & No & .00001 & .00003 & .00001 & No & Spearman \\
\hline C11 & Intervalo & 1 & $-0,139$ & $-0,191$ & No & .00567 & .00103 & .00022 & No & Spearman \\
\hline $\mathrm{C} 12$ & Intervalo & 0 & $-0,227$ & $-0,288$ & No & .46832 & .00030 & .05279 & $\mathrm{Si}$ & Spearman \\
\hline $\mathrm{C} 13$ & Intervalo & 1 & $-0,060$ & $-0,135$ & No & .17289 & .00070 & .00002 & No & Spearman \\
\hline C14 & Intervalo & 0 & $-0,252$ & $-0,216$ & No & .00006 & .00002 & .00009 & No & Spearman \\
\hline $\mathrm{C} 15$ & Intervalo & 1 & $-0,326$ & $-0,300$ & No & .00045 & .00010 & .00017 & No & Spearman \\
\hline $\mathrm{C} 16$ & Intervalo & 1 & $-0,244$ & $-0,185$ & No & .00003 & .00028 & .00021 & No & Spearman \\
\hline $\mathrm{C} 17$ & Intervalo & 1 & $-0,032$ & $-0,022$ & No & .00026 & .00008 & .00001 & No & Spearman \\
\hline $\mathrm{C} 18$ & Intervalo & 0 & $-0,111$ & $-0,102$ & No & .00076 & .00058 & .00051 & No & Spearman \\
\hline $\mathrm{C} 19$ & Intervalo & 0 & $-0,244$ & $-0,245$ & No & .00066 & .00018 & .00041 & No & Spearman \\
\hline $\mathrm{C} 20$ & Intervalo & 1 & $-0,224$ & $-0,200$ & No & .00001 & .00004 & .00005 & No & Spearman \\
\hline $\mathrm{C} 21$ & Intervalo & - & - & - & - & .00009 & .00001 & .00757 & No & Spearman \\
\hline $\begin{array}{l}\text { Nota: C01: } \\
\text { C06: Apren } \\
\text { Ingles. C1 } \\
\text { Ingles. C16 }\end{array}$ & $\begin{array}{l}\text { e Activo } \\
\text { xivo vs L } \\
\text { aje Teóri } \\
\text { je Pragm }\end{array}$ & a C & $\begin{array}{l}\text { : Apren } \\
\text { rrelaci }\end{array}$ & $\begin{array}{l}\text { nático, } \\
\text { ada de }\end{array}$ & $\begin{array}{l}\text { zaje I } \\
\text { endiza }\end{array}$ & $\begin{array}{l}\text { órico vs } \\
\text { vs Social } \\
\text { miento ac }\end{array}$ & $\begin{array}{l}\text { 14: Apre } \\
\text { prendizaj } \\
\text { n las prue }\end{array}$ & $\begin{array}{l}\text { eórico vs } \\
\text { tico vs Na } \\
11 .\end{array}$ & $\begin{array}{l}\text { Aprer } \\
\text { C10: } \\
\text { C15: } \\
\text { 20: } \mathrm{Al}_{1}\end{array}$ & $\begin{array}{l}\text { ctivo vs Ingles } \\
\text { aje Reflexivo } \\
\text { aje Teórico vs } \\
\text { Pragmático }\end{array}$ \\
\hline
\end{tabular}

Nótese que la presencia de datos atípicos se evidenció en diez de las 20 combinaciones, mientras que la linealidad y la normalidad solo pudieron constatarse, respectivamente, en ningún caso y en dos de tales pares, pero de forma no concurrente. Este hecho sustenta la afirmación inicial por cuanto no se cumplen de manera simultánea las condiciones para emplear la correlación de Pearson.

La tabla 7, muestra los resultados de este punto exhibiendo relaciones significativas en seis de los pares. De manera específica, se observaron asociaciones positivas en las combinaciones de Ciencias naturales vs estilo reflexivo vs teórico y de carácter negativo con el estilo activo. También se denotó correlación positiva entre la combinación de sociales vs estilo teórico y negativo con el estilo activo. El estilo pragmático se correlaciona positivamente con la competencia de inglés.

Tabla 7. Correlación: Estilos De Aprendizaje y Rendimiento Académico

\begin{tabular}{ccccccc}
\hline \multicolumn{2}{c}{ Estilos de Aprendizaje } & Lectura Critica & Matemáticas & Sociales & Ciencias Naturales & Inglés \\
\hline \multirow{2}{*}{ Activo } & Rho & $-0,142$ & $-0,022$ &,$- 179^{*}$ &,$- 219^{*}$ & 0,049 \\
& Valor-P & 0,102 & 0,805 & 0,039 & 0,011 & 0,575 \\
\multirow{2}{*}{ Reflexivo } & Rho & 0,075 & 0,092 & 0,067 &, $272^{* *}$ & 0,097 \\
& Valor-P & 0,388 & 0,294 & 0,441 & 0,002 & 0,268 \\
\multirow{2}{*}{ Teórico } & Rho & $-0,009$ & 0,065 &, $212^{*}$ &, $181^{*}$ & 0,105 \\
& Valor-P & 0,915 & 0,458 & 0,014 & 0,037 & 0,229 \\
\multirow{2}{*}{ Pragmático } & Rho & $-0,096$ & 0,027 & 0,061 & $-0,015$ &, $225^{* *}$ \\
& Valor-P & 0,272 & 0,755 & 0,488 & 0,865 & 0,009 \\
& & **. La correlación es significativa en el nivel 0,01 (bilateral). & & \\
\end{tabular}


De acuerdo a la tabla 1 y 2 , el estilo reflexivo perfila a nuestros estudiantes con características de ponderados, concienzudos, receptivos, analíticos y exhaustivos, a los estudiantes reflexivos, les gusta considerar las experiencias y observarlas desde diferentes respectivas. Su filosofía según Honey y Mumford (1986), consiste en ser prudentes, no dejar piedra sin mover, mirar bien antes de pasar. Son personas que gustan considerar todas las alternativas antes de realizar un movimiento. Según Catalina A. y Gallego D. y Honey (1994), "las personas que tienen preferencias por este estilo tienen puntuaciones elevada en el estilo activo" (p. 87), situación que no se cumple en esta investigación ya que el estilo segunda valoración promedial fue el pragmático, pero de acuerdo a las consideraciones establecidas por los que creadores del instrumento hay una correlación débil entre estos dos estilos, que también se presenta en la matriz del test LSQ.

Al analizar el baremo general, con los resultados obtenidos de la tabla 3 , se denotan valores diferentes a los propuestos por P. Honey y A. Mumford (Op. cit), en donde la preferencia muy baja, la han puntuado menos del 10\% de la muestra exceptuando el estilo reflexivo con valor muy próximo. En este sentido el porcentaje muestral más bajo en la preferencia muy baja lo obtuvo el estilo teórico, seguido del estilo pragmático y el activo. El estilo reflexivo para esta preferencia tiene la representación muestral más alta ( $9 \%)$. El nivel de preferencia bajo, para los estilos de aprendizaje activo, pragmático y teórico, está muy por debajo del $20 \%$ de la muestra, propuesto por P. Honey y A. Mumford (Op. cit), al igual que la preferencia anterior se mantiene la tendencia en donde el estilo activo tiene el porcentaje muestral más bajo $(9,8 \%)$, y el estilo reflexivo el más alto (18\% muestral). Por otra parte, la preferencia moderada, la puntúan el $40 \%$ de la población en los estilos activo y teórico, en el estilo reflexivo se encuentra más de la mitad de los estudiantes, el estilo pragmático no alcanza el umbral del $40 \%$ muestral. La frecuencia muy alta supera las expectativas en los estilos teórico, activo y pragmático, superando el porcentaje del 10\% muestral.

Es preciso mencionar que estos cuatro niveles de desempeño manifestados en el gráfico 4, solo aplican para las pruebas de lectura crítica, matemáticas, ciencias naturales y sociales, ya que para la prueba de inglés hay otros niveles de desempeño estipulados y categorizados. Según el Ministerio de Educación Nacional (2016), "estos niveles describen lo que saben y saben hacer los estudiantes en una determinada prueba del examen de acuerdo a las especificaciones definidas en los estándares básicos de competencias establecidos por el Ministerio de Educación Nacional" (p .24). De esta forma se pueden analizar las fortalezas y debilidades de los estudiantes, respecto a cada una de las competencias y tomar acciones de mejora para los periodos siguientes.

Respecto a la tabla 7, el estilo activo presenta una asociación significativa, de carácter negativo, con el rendimiento académico en las pruebas saber 11, de sociales y ciencias naturales a un nivel de significancia de 0,05 (bilateral). Por otra parte, el rendimiento académico en la prueba de ciencias naturales, presenta asociación significativa, con el estilo de aprendizaje reflexivo y teórico, con un nivel de significancia de 0,01 y 0,05 respectivamente.

El estilo teórico, manifiesta correlación significativa, de carácter positivo con el rendimiento académico en la prueba de sociales. Por último, el estilo de aprendizaje pragmático, presenta asociación a nivel 0.01 bilateral con el rendimiento académico en la prueba saber 11 de inglés.

\section{Conclusiones}

Partiendo de los análisis descriptivos y correlacional de las variables, se concluye que hay una tendencia hacia el estilo de aprendizaje reflexivo y pragmático, en cada una de las instituciones educativas, además los hombres tienen mayores puntuaciones para los estilos activo, teórico, y pragmático. La preferencia moderada contiene la mayor parte de la muestra tanto en hombre como 
mujeres, en cada uno de los estilos. Por otra parte se identificó los niveles de desempeño, de cada una de las pruebas saber 11, manifestando que los niveles 1 y 2 son nulos, en cada una de las competencias. La prueba con mejor promedio fue la de matemática y la ultima la de sociales, con un nivel de desempeño 2 muy alto. La prueba de inglés, manifiesta que la mayoría de la muestra tiene un nivel de desempeño A2, y un porcentaje mínimo tiene el máximo nivel o $\mathrm{B}+$; el nivel A-, es nulo.

De igual manera se evidenció que existe una relación significativa de carácter positivo, entre el estilo de aprendizaje reflexivo y el rendimiento académico en la prueba de ciencias naturales, situación que similar con el estilo de aprendizaje teórico. Por otra parte, el rendimiento académico en la prueba mencionada y en la prueba de sociales, manifiestan correlación baja de carácter negativo con el estilo activo. El estilo pragmático se asocia positivamente con el rendimiento en la competencia de inglés.

\section{Referencias}

Alonso, C. (1992). Análisis y Diagnóstico de los Estilos de Aprendizaje en Estudiantes Universitarios. Tomo I. Madrid: Colección Tesis Doctorales. Editorial de la Universidad Complutense.

Camarero, et al (2000). Estilos y estrategias de aprendizaje en estudiantes universitarios. Psicothema, 12, 615-622.

Castaño, G. y Calles, A. (2006). Independencia de los estilos de aprendizaje de las variables cognitivas y afectivo motivacionales. Universidad Complutense de Madrid.

Catalina A. y Gallego D. y Honey (1994). los estilos de aprendizaje, procedimientos de diagnóstico y mejora. Ediciones mensajero S.A. Bilbao, Madrid

Grupo Educativo Milton Ochoa (2016). Ranking de los mejores colegios 2016. Revista en línea]. Recuperado: https://miltonochoa.com.
co/home/Ranking/Ranking\%20Calendario $\% 20$ A\%20(2016)/PDF/Ponderado\%20Publicas.pdf.

Guild y Garger (1998)Marching to different drummers. Virginia, USA: ASCD asociación for supervisión and Curriculum development. 2nd editon.

Hernández, C. A., Prada, R., \& Rincón, G. A. (2018). Inteligencias múltiples y rendimiento académico del área de matemáticas en estudiantes de educación básica primaria. Infancias Imágenes, 17(2), 163-175.

Hernández-Dávila, R., \& Díaz-Abdala, W. E. (2017). Consideraciones teóricas y metodológicas para investigar sobre deserción escolar. Revista Perspectivas, 2(2), 108-119.

Honey, P. y Munford, A. (1986). Using your learning styles. Maidenhead: Peter Honey.

Keefe, J.W. (1988). Profiling and Utilizing Learning Style. Reston Virginia: National Association of Secondary School Principals.

Lozano, A. (2000). Estilos de Aprendizaje y Enseñanza. Un panorama de la estilística educativa. ITESM Universidad Virtual - ILCE. México: Trillas.

Ministerio de Educación Nacional de Colombia (2016). Guía de interpretación y uso de resultados del examen Saber 11. Recuperado: https://www.google.com.co/

Revista Dinero (2016). Los mejores colegios de Colombia según las pruebas saber 11. [Revista en línea]. Recuperado: http://www. dinero.com/contenidos-editoriales/especialde-colegios-2016/multimedia/rankingde-los-mejores-colegios-de-colombia-enel-2016/222439.

Varela Hincapié, M.(2014). Relación Entre Los Estilos De Aprendizaje Y Los Niveles De Creatividad Motriz En Los Estudiantes De La Institución EducativaLasDeliciasDelMunicipioDeElBagre. Tesis De Maestría no publicada. Universidad De Antioquia. Recuperado: http://tesis. udea.edu.co/bitstream/10495/2774/1/1.\%20 PROYECTO $\% 20$ MAESTR \%C3\%8DA \%20 EN\%20EDUCACI\%C3\%93N\%20FINAL. 